J Phys Chem Lett. 2016 March 17; 7(6): 944-949. doi:10.1021/acs.jpclett.6b00137.

\title{
Constant pH Molecular Dynamics Reveals pH-modulated Binding of Two Small-Molecule BACE1 Inhibitors
}

\author{
Christopher R. Ellis ${ }^{1, \S}$, Cheng-Chieh Tsai ${ }^{1, \S}$, Xinjun Hou${ }^{2}$, and Jana Shen ${ }^{1, *}$ \\ ${ }^{1}$ Department of Pharmaceutical Sciences, University of Maryland School of Pharmacy, Baltimore, \\ MD \\ ${ }^{2}$ Neuroscience Worldwide Medicinal Chemistry, Pfizer Worldwide Research and Development, \\ Cambridge, MA
}

\section{Abstract}

Targeting BACE1 with small-molecule inhibitors offers a promising route for treatment of Alzheimer's disease. However, the intricate $\mathrm{pH}$ dependence of BACE1 function and inhibitor efficacy has posed major challenges for structure-based drug design. Here we investigate two, structurally similar BACE1 inhibitors that have dramatically different binding affinity and inhibitory activity using continuous constant $\mathrm{pH}$ molecular dynamics (CpHMD). At high $\mathrm{pH}$, both inhibitors are stably bound to BACE1, however, within the enzyme active $\mathrm{pH}$ range, only the iminopyrimidinone-based inhibitor remains bound, while the aminothiazine-based inhibitor becomes partially dissociated following the loss of hydrogen bonding with the active site and change of the 10s loop conformation. The drastically lower affinity of the second inhibitor is due to the protonation of a catalytic aspartate and the lack of a propyne tail. This work demonstrates that $\mathrm{CpHMD}$ can be used for screening $\mathrm{pH}$-dependent binding profiles of small-molecule inhibitors, providing a new tool for structure-based drug design and optimization.

$\beta$-secretase (BACE1), a leading therapeutic target for treating Alzheimer's disease (AD), is one of the two aspartic proteases that cleave the amyloid precursor protein to produce the $\beta$ amyloid peptide $(\mathrm{A} \beta)$. Subsequent oligomerization and fibrillation of $\mathrm{A} \beta$ lead to neuritic plaques which are linked to the onset of AD. ${ }^{1}$ Since the discovery of BACE1 over a decade ago, hundreds of inhibitors have been developed, however, none have passed the FDA required clinical trial process and entered the market. ${ }^{2}$ The first generation of designed inhibitors are peptide-like transition-state analogs, which demonstrated excellent in vitro binding affinity for BACE1, however, high molecular weight, polarity and flexibility as well as numerous hydrogen bond donors and acceptors reduce their ability to penetrate the bloodbrain barrier (BBB) to reach the central nervous system where BACE1 resides. ${ }^{3}$ Therefore, in more recent years, small-molecule inhibitors have emerged as a promising route for treatment and prevention of $\mathrm{AD}{ }^{3}$

Corresponding Author: jshen@rx.umaryland.edu.

§oint first authors.

Supporting Information

Details of the computational protocols and additional analyses. This material is available free of charge via the Internet at http:// pubs.acs.org. 
BACE1 is a monomeric protein localized in the acidic endosome and trans-Golgi apparatus. ${ }^{2}$ The catalytic domain of BACE1 contains a catalytic dyad, Asp32 and Asp228, which respectively act as a generalized acid and base to catalyze peptide hydrolysis. ${ }^{4}$ Three key regions in the structure of BACE1 must be considered when designing inhibitors (Figure 1A). First, the aspartic dyad directly interacts with inhibitors via hydrogen bonds and/or salt bridges. Generally, peptidomimetic compounds contain a hydroxyl group that forms a hydrogen bond with the active site, while small-molecule inhibitors contain a basic amine site with a $\mathrm{p} K_{\mathrm{a}}$ greater than 6 that forms a salt bridge/hydrogen bond with one of the catalytic aspartates. Second, the flap, a $\beta$-hairpin loop spanning residues Tyr68 to Glu77, lies directly over the catalytic dyad and can open and close at room temperature to allow substrate and inhibitor access to the active site. ${ }^{3,5}$ Finally, the 10s loop, a $\beta$-hairpin loop with Ser10 at the tip, can adopt an "up" or "down" conformation, modulating the accessibility of an additional binding pocket, which further impacts the inhibitor effectiveness. ${ }^{6}$

Recently, heterocycles that contain a basic amine site, positively charged in the enzyme active $\mathrm{pH}$ range ${ }^{9}$ to engage the negatively charged aspartyl dyad, have emerged as a leading scaffold for designing potent, selective BACE1 inhibitors. ${ }^{3}$ These heterocycles are often referred to as the aspartyl binding motif (ABM). However, determining the optimal $\mathrm{p} K_{\mathrm{a}}$ of the basic amine has been a formidable task. While very high basicity $\left(\mathrm{p} K_{\mathrm{a}}>9\right)$ increases the likelihood of the salt-bridge formation with the aspartyl dyad, it decreases brain permeability and thus lowers in vivo efficacy. ${ }^{10,11}$ Conversely, inhibitors with low basicity $\left(\mathrm{p} K_{\mathrm{a}} \sim 5.5\right)$, although more able to cross the BBB, are not active under mildly acidic conditions (such as pH 6.5). ${ }^{12}$

In this work we investigate two potent drug-like BACE1 inhibitors. Inhibitor $1^{7}$ closely resembles the Merck inhibitor MK8931 currently under phase 2/3 clinical trial, ${ }^{2}$ while inhibitor 2 was discovered by Eli Lilly and tested in preclinical animal models and humans but discontinued due to retinal toxicity. ${ }^{8}$ Recently, structure-based optimization has been applied to 2 by Pfizer in an effort to reduce toxicity. ${ }^{13,14}$ Both inhibitors occupy the same binding pockets and more importantly, they contain a six-membered ABM that forms bidentate hydrogen bond interactions with the catalytic dyad via the endo- and exocyclic amines (Figure 2A and 2B). Note that, with the $\mathrm{p} K_{\mathrm{a}}$ 's of 7.5 and 8.7 for $\mathbf{1}$ and $\mathbf{2}$, respectively (Figure 1B and 1C), the endocyclic amines are charged in the BACE1 active pH range. ${ }^{9,15}$ Interestingly, despite having similar structure and nearly identical binding modes, the in vitro binding affinity and in vivo inhibitory activity of the two inhibitors are dramatically different. The $\mathrm{IC}_{50}$ values of $\mathbf{2}$ is 30 fold higher than $\mathbf{1}$ (Figure $1 \mathrm{~B}$ and $1 \mathrm{C}$ ). Curiously, however, the $\mathrm{p} K_{\mathrm{a}}$ of $\mathbf{2}$ is 1.2 units higher than that of $\mathbf{1}$, which, according to the current consensus based on chemical intuition, ${ }^{12,3}$ should make 2 a stronger binder. Thus, there is no intuitive explanation for the significantly lower affinity of $\mathbf{2}$.

To explore the interactions of the two inhibitors with BACE1 in the enzyme active $\mathrm{pH}$ range, we performed constant $\mathrm{pH}$ molecular dynamics (pHMD) simulations starting from the crystal structures of BACE1 complexed with 1 and $\mathbf{2}$ (PDB ID 4FRS ${ }^{7}$ and 4 YBI ${ }^{8}$, respectively). Previous pHMD simulations and Born approximation methods have been utilized to predict shifts in the BACE1 catalytic dyad $\mathrm{p} K_{\mathrm{a}}$ with neutral peptide-like 
inhibitors, while this work considers charged small molecules that change protonation state as a function of $\mathrm{pH} .{ }^{16,17,5,18}$ Based on discrete ${ }^{19,20,21,22}$ or continuous variables ${ }^{23,24,25}$ to represent protonation states, pHMD enables ionizable sites of proteins and/or other solutes to simultaneously change protonation states in response to conformational dynamics at a specified solution $\mathrm{pH}$, thus extending the commonly simulated NPT or NVT ensemble to include a constant $\mathrm{pH}$ condition. Here we employed continuous constant $\mathrm{pH}$ molecular dynamics (CpHMD) ${ }^{23,24}$ with a hybrid-solvent scheme and $\mathrm{pH}$ replica-exchange. ${ }^{26}$ The hybrid-solvent $\mathrm{CpHMD}$ method makes use of accurate conformational sampling in explicit solvent and efficient calculation of solvation free energies with the generalized Born implicit-solvent model. ${ }^{26}$ Combined with the $\mathrm{pH}$ replica-exchange sampling protocol, ${ }^{26}$ it offers accurate $\mathrm{p} K_{\mathrm{a}}$ convergence on the order of $1 \mathrm{~ns}$ per $\mathrm{pH}$ replica, ${ }^{26}$ in particular for interior sites of proteins that are notoriously challenging to predict with static structurebased electrostatics methods. ${ }^{27,28} \mathrm{~A}$ benchmark study showed that hybrid-solvent CpHMD can predict the $\mathrm{p} K_{\mathrm{a}}$ 's of enzyme catalytic sites with a correct order and accuracy of about 1 pH unit. ${ }^{26}$ Most recently, we applied hybrid-solvent CpHMD to study the apo form of BACE1, revealing a $\mathrm{pH}$-dependent population shift between three conformational states that gives rise to the bell-shaped $\mathrm{pH}$ profile of the enzymatic activity. In this work, we demonstrate that subtle differences in the titration behavior of the BACE1-inhibitor complex can lead to drastically different binding behavior within the enzyme active $\mathrm{pH}$ range. Such knowledge has been lacking although it is crucial for the design and optimization of BACE1 inhibitors.

BACE1 functions within a narrow $\mathrm{pH}$ range (3.5-5.5) ${ }^{9,15}$ and hydrolyzes peptide bonds via a general acid-base mechanism. ${ }^{3}$ The experimentally determined $\mathrm{p} K_{\mathrm{a}}$ 's of the acidic and basic components of the catalytic dyad are 5.2 and 3.5, respectively. ${ }^{4}$ Therefore, within the BACE1 active $\mathrm{pH}$ range, the catalytic dyad is likely to be in the monoprotonated state. Our previous simulation of apo BACE1 using the hybrid-solvent CpHMD gave the calculated $\mathrm{p} K$ a of 4.1 for Asp32 and 1.9 for Asp228, about one $\mathrm{pH}$ unit below the experimentally determined values. ${ }^{5}$ Accordingly, the simulated $\mathrm{pH}$ range of BACE1 where the catalytic dyad is in the monoprotonated state is $2.5-4.5$ in the simulation. To account for the systematic deviation, we will adopt the simulated active $\mathrm{pH}$ range in the remainder of the discussion. Importantly, adoption of the simulated active $\mathrm{pH}$ range instead of the experimentally determined active $\mathrm{pH}$ range does not impact the results. The simulated active $\mathrm{pH}$ range is used as a guide for the simulated $\mathrm{pH}$ condition that likely represents the active form of BACE1.

We first consider the protonation states of Asp32 and Asp228. The active site of BACE1 is mostly hydrophobic and no charged or titratable residues are with $8 \AA$ of the dyad, therefore, shifts in the catalytic dyad $\mathrm{p} K_{\mathrm{a}}$ 's are predominately effected by the positive charge of the inhibitors. When BACE1 is bound to a basic inhibitor, the $\mathrm{p} K_{\mathrm{a}}$ of Asp32 is expected to shift down as the hydrogen bond/salt bridge with the positively charged endocyclic amine stabilizes the charged state. This is indeed the case when BACE1 is bound with 1: the $\mathrm{p} K_{\mathrm{a}}$ of Asp32 is lowered significantly from the apo-state value of 4.1 to 2.5 (Figure 2C, blue). However, binding with 2 produces a negligible $\mathrm{p} K_{\mathrm{a}}$ shift: the $\mathrm{p} K_{\mathrm{a}}$ is lowered only by 0.4 unit to 3.7 (Figure $2 \mathrm{C}$, red). Thus, in the active $\mathrm{pH}$ range, Asp32 becomes deprotonated in 
the presence of $\mathbf{1}$; however, it remains partially protonated in the presence of $\mathbf{2}$. In contrast to Asp32, neither inhibitor significantly impacts the titration behavior of Asp228: the $\mathrm{p} K_{\mathrm{a}}$ 's are 2.3 and 2.0 in the presence of $\mathbf{1}$ and 2, respectively (Table S1). Thus, Asp228 remains completely deprotonated in the active $\mathrm{pH}$ range. This indicates that the hydrogen bond between Asp228 and the neutral exocyclic amine group of the two inhibitors has approximately the same strength.

Next, we examine the most important BACE1-inhibitor interactions, i.e., the hydrogen bonds between the catalytic aspartates and the endo- and exocyclic amine groups on the ABM. Above the active $\mathrm{pH}$ range, both inhibitors form stable hydrogen bonds with the aspartyl dyad, as evident from the high percentage of hydrogen bond occupancy (Figure 2D). The hydrogen bond between the endocyclic amine group and Asp32 is particularly strong, as it is also a salt bridge due to the charge on both groups. However, within the active $\mathrm{pH}$ range, the occupancy of the hydrogen bonds between $\mathbf{2}$ and the aspartate dyad decreases sharply as $\mathrm{pH}$ is lowered (Figure 2D, red). In contrast, the hydrogen bonds between $\mathbf{1}$ and the catalytic dyad remain almost completely intact within the active $\mathrm{pH}$ range (Figure 2D, blue).

Interestingly, the shape of the $\mathrm{pH}$-dependent hydrogen bond occupancy is nearly identical as the $\mathrm{pH}$-dependent unprotonated fraction of Asp32 in the active $\mathrm{pH}$ range. Note, Asp228 is always deprotonated in this $\mathrm{pH}$ range. Thus, the data suggests that, in the presence of $\mathbf{2}$, Asp32 becomes protonated in the active $\mathrm{pH}$ range, destabilizing the hydrogen bond with the inhibitor. As expected, the hydrogen bond occupancy is correlated with the hydration number of the active site. Within the active $\mathrm{pH}$ range, breakage of the hydrogen bond between 2 and Asp32 leads to entrance of water molecules to the active site (Figure 2E, red). In contrast, when BACE1 is complexed with $\mathbf{1}$, the active site remains dry in the entire active $\mathrm{pH}$ range (Figure $2 \mathrm{E}$, blue).

The breakage of the hydrogen bonds and entrance of water into the active site is an indication of dissociation of inhibitor $\mathbf{2}$ from BACE1. To quantify the degree of dissociation, we calculated the fraction of bound configurations as a function of $\mathrm{pH}$. A bound configuration is considered formed when the distance between the endocyclic nitrogen of the ABM and the carbonyl carbon of Asp32 is less than $4 \AA$. Above the active $\mathrm{pH}$ range, both inhibitors are largely bound (Figure $3 \mathrm{~A}$ and $3 \mathrm{C}$ ). However, in the active $\mathrm{pH}$ range, only $\mathbf{1}$ remains bound, while the fraction of bound configurations in the presence of 2 sharply decreases as $\mathrm{pH}$ is decreased. At the most active $\mathrm{pH}$ (3.5) of BACE1, less than $25 \%$ of 2 is bound (Figure 3B and 3C). The pH-induced dissociation of 2 is a consequence of the protonation of Asp32 and offers an explanation for the significantly reduced binding affinity and activity of $\mathbf{2}$ when measured under the most active $\mathrm{pH}$ condition of the enzyme. ${ }^{7,8} \mathrm{We}$ note that, since dissociation of the inhibitor occurs on a longer time scale compared to our limited simulation length, the fraction of unbound configurations shown in Figure $3 \mathrm{C}$ is likely not quantitative. However, a quantitative prediction of binding affinity is not in the scope of the current work as the simulation length required for absolute convergence of the entire ensemble of unbound configurations is prohibitively expensive. Rather, our focus here is to provide a method for screening $\mathrm{pH}$-dependent binding profiles of small-molecule inhibitors. Importantly, within the $18 \mathrm{~ns}$ of simulation, individual replicas undergo unbinding 
and binding events as they walk through $\mathrm{pH}$ space, this would likely be missed by performing conventional MD simulations with fixed protonation states.

Examination of the ABM suggests two possible factors contributing to the different protonation behavior of Asp32 in the presence of $\mathbf{1}$ and $\mathbf{2}$. First, the presence of the exocyclic methyl group in the ABM of $\mathbf{1}$ provides steric hindrance for water to access the active site, thus stabilizing the hydrogen bonds between the endo- and exocyclic amine groups and Asp32 (Figure 2A, blue line) and making the deprotonated state of Asp32 favorable. Second, the partial negative charge $(-0.33)$ of the sulfur atom on the ABM of 2 (6 $\AA$ from Asp32, Figure 2B) makes the deprotonation of Asp32 unfavorable. In contrast, at the same position in the ABM of $\mathbf{1}$, the methyl amine group is nearly neutral $(+0.02)$ with a positive partial charge on the methyl group $(+0.11)$. Thus, despite the fact that inhibitor $\mathbf{1}$ is less basic (measured $\mathrm{p} K_{\mathrm{a}}$ of 7.5) than 2 (measured $\mathrm{p} K_{\mathrm{a}}$ of 8.7), the $\mathrm{p} K_{\mathrm{a}}$ of Asp32 is shifted lower in the presence of $\mathbf{1}$, which makes the catalytic dyad di-deprotonated in the active $\mathrm{pH}$ range and enhances the electrostatic attraction with the positively charged inhibitor. Notably, neither inhibitor interacts with other charged or titratable residue in both the bound and unbound configurations.

Finally, we test a hypothesis that correlates the position of the 10s loop with inhibitor efficacy. Surveys of inhibitor-bound BACE1 crystal structures showed that the 10s loop adopts either "up" or "down" conformation. ${ }^{6}$ In the 10s-down conformation, Ser10 can form a hydrogen bond with Thr232 (Figure 4A), while in the 10s-up conformation, Ser10 is distant from Thr232 (Figure 4B). Based on a series of tertiary carbinamine derived compounds, McGaughey et al. hypothesized that inhibitors that form favorable interactions with Thr232 may disrupt the hydrogen bond between Thr232 and Ser10, effectively locking the 10s loop in the up configuration and increasing the binding interactions. ${ }^{6}$

To test this hypothesis, we calculated the hydrogen bond occupancy of Thr232 with Ser10 and the inhibitors. In the simulation of BACE1 complexed with $\mathbf{1}$, the pyridine ring of the inhibitor can not reach Thr232 due to the steric hindrance by the alkyne tail and does not modulate the 10s conformation. In contrast, in the simulation of BACE1 complexed with $\mathbf{2}$, the lack of alkyne group allows its pyrimidine ring to approach Thr232 in a pH-dependent manner. Above the enzyme active $\mathrm{pH}$ range, Thr232 can form a hydrogen bond with Ser10 (about 25\%) but not with $\mathbf{2}$ (Figure 4C), and 10s loop is mostly down, consistent with the Xray crystal structure, which was obtained at $\mathrm{pH} 7.4$ (PDB ID 4YBI). Interestingly, as $\mathrm{pH}$ decreases in the active $\mathrm{pH}$ range, the hydrogen bond occupancy for Ser10 sharply decreases, whereas that for the inhibitor sharply increases until it plateaus at around $\mathrm{pH} 3.5$, corresponding to the most active $\mathrm{pH}$ of BACE1 (Figure 4C). The formation of the latter hydrogen bond can be attributed to the protonation of the pyrimidine nitrogen (Figure 4D), enabling it to donate a hydrogen to Thr232 effectively locking the 10s loop in the up configuration (Figure 4B). Thus, together with the earlier analysis showing that 2 becomes partially detached from BACE1 in the active $\mathrm{pH}$ range, these data support the hypothesis that inhibitors that compete with Ser10 to form a hydrogen bond with Thr232 decrease binding interactions with the catalytic dyad. 
In summary, constant $\mathrm{pH}$ molecular dynamics simulations revealed that, despite having a similar structure and nearly identical binding modes, inhibitors $\mathbf{1}$ and $\mathbf{2}$ exhibit different $\mathrm{pH}$ dependent interactions with BACE1. Within the enzyme active $\mathrm{pH}$ range, $\mathbf{2}$ shows disrupted hydrogen bonds with the catalytic dyad in favor of hydrogen bond with Thr232. The former is due to the protonation of Asp32, which decreases the attraction to the charged aminothiazine ring. The hydrogen bonding with Thr232 is due to the lack of a propyne tail (present in 1), which allows the pyrimidine ring to move closer and donate a hydrogen from the protonated amine effectively forcing the 10s loop to the up position. Together, these changes lead to a significantly lowered binding affinity of $\mathbf{2}$ in the BACE1 active $\mathrm{pH}$ range, consistent with the drastically higher $\mathrm{IC}_{50}$ value.

Our work demonstrates how mutual interactions between BACE1 and inhibitors result in subtle changes in the protonation states which in turn alters the binding interactions and strength. Thus, obtaining a detailed knowledge of the $\mathrm{pH}$-dependent conformational dynamics and protonation-state changes of protein-small molecule complexes is crucial for designing potent, selective inhibitors of BACE1 and other enzymes that contain titratable catalytic sites and function over a narrow $\mathrm{pH}$ range. CpHMD can be used for illuminating $\mathrm{pH}$-dependent dynamic details and screening $\mathrm{pH}$ profiles of small-molecule binding, thus providing a unique new tool for structure-based drug design and optimization.

\section{METHODS AND PROTOCOLS}

Continuous constant $\mathrm{pH}$ molecular dynamics (CpHMD) simulations ${ }^{23,24}$ with a hybridsolvent scheme and $\mathrm{pH}$ replica-exchange protocol ${ }^{26}$ were performed on BACE1 in complex with inhibitors 1 and $\mathbf{2}$ starting from the respective crystal structures (PDB ID 4FRS ${ }^{7}$ and $4 \mathrm{YBI}^{8}$ ) using CHARMM (version C37b). ${ }^{29}$ The CHARMM22/CMAP all-atom force field ${ }^{30,31}$ was used to represent BACE1, while the force field parameters for the inhibitors were obtained in house following the protocol of CHARMM General Force Field $(\mathrm{CGenFF})^{32}$. Each system was simulated using $20 \mathrm{pH}$ replicas in the $\mathrm{pH}$ range 1.3-8. Each BACE1 complex was simulated for $26 \mathrm{~ns}$ per replica under NPT conditions, resulting in a total simulation time of $520 \mathrm{~ns}$. The last $18 \mathrm{~ns}$ per replica (360 ns in total) were used for analysis. Other simulation parameters and setting were identical to those in our previous work ${ }^{5}$. The titratable sites include all Asp, Glu and His residues on the protein and the amine group on the pyridine/pyrimidine ring of the inhibitor (see Table S1 for all calculated $\mathrm{p} K_{\mathrm{a}}$ 's). The model $\mathrm{p} K_{\mathrm{a}}$ 's for Asp, Glu and His are 4.0, 4.4, and 6.5, respectively, ${ }^{26}$ while those for inhibitor $\mathbf{1}$ and $\mathbf{2}$ are 2.9 and 3.7, respectively. The latter $\mathrm{p} K_{\mathrm{a}}$ 's were estimated using the program MoKa. ${ }^{33}$ The $\mathrm{p} K_{\mathrm{a}}$ measurements for the inhibitors were conducted using capillary electrophoresis experiment following the protocol from ref. ${ }^{34}$

\section{Supplementary Material}

Refer to Web version on PubMed Central for supplementary material. 


\section{Acknowledgments}

We wish to thank Fang-Yu Lin and Kenno Vanommeslaeghe who developed the force fields of the small-molecule inhibitors. Financial support is provided by National Science Foundation (MCB1305560) and National Institutes of Health (GM098818).

\section{References}

1. Lansbury PT, Lashuel HA. Nature. 2006; 443:774-779. [PubMed: 17051203]

2. Vassar R. Alzheimer's Res Ther. 2014; 6:89. [PubMed: 25621019]

3. Yuan J, Venkatraman S, Zheng Y, McKeever BM, Dillard LW, Singh SB. J Med Chem. 2013; 56:4156-4180. [PubMed: 23509904]

4. Toulokhonova L, Metzler WJ, Witmer MR, Copeland RA, Marcinkeviciene J. J Biol Chem. 2003; 278:4582-4589. [PubMed: 12458195]

5. Ellis CR, Shen J. J Am Chem Soc. 2015; 137:9543-9546. [PubMed: 26186663]

6. McGaughey GB, Colussi D, Graham SL, Lai MT, Munshi SK, Nantermet PG, Pietrak B, Rajapakse HA, Selnick HG, Stauffer SR, Holloway MK. Bioorg Med Chem Lett. 2007; 17:1117-1121. [PubMed: 17112725]

7. Stamford AW, et al. ACS Med Chem Lett. 2012; 3:897-902. [PubMed: 23412139]

8. May PC, et al. J Neurosci. 2011; 31:16507-16516. [PubMed: 22090477]

9. Shimizu H, Tosaki A, Kaneko K, Hisano T, Sakurai T, Nukina N. Mol Cell Biol. 2008; 28:36633671. [PubMed: 18378702]

10. Swahn BM, et al. Bioorg Med Chem Lett. 2012; 22:1854-1859. [PubMed: 22325942]

11. Hilpert H, et al. J Med Chem. 2013; 56:3980-3995. [PubMed: 23590342]

12. Stachel SJ, et al. Bioorg Med Chem Lett. 2009; 19:2977-2980. [PubMed: 19409780]

13. Butler CR, et al. J Med Chem. 2015; 58:2678-2702. [PubMed: 25695670]

14. Brodney MA, et al. J Med Chem. 2015; 58:3223-3252. [PubMed: 25781223]

15. Grüninger-Leitch F, Schlatter D, Küng E, Nelböck P, Döbeli H. J Biol Chem. 2002; 277:46874693. [PubMed: 11741910]

16. Kim MO, Blachly PG, McCammon JA. PLoS Comput Biol. 2015; 11:e1004341. [PubMed: 26506513]

17. Polgar T, Keseru GM. J Med Chem. 2005; 48:3749-3755. [PubMed: 15916426]

18. Domnguez JL, Christopeit T, Villaverde MC, Gossas T, Otero JM, Nystrom S, Baraznenok V, Lindstrom E, Danielson UH, Sussman F. Biochemistry. 2010; 49:7255-7263. [PubMed: 20687525]

19. Baptista AM, Teixeira VH, Soares CM. J Chem Phys. 2002; 117:4184-4200.

20. Mongan J, Case DA, McCammon JA. J Comput Chem. 2004; 25:2038-2048. [PubMed: 15481090]

21. Swails JM, Roitberg AE. J Chem Theory Comput. 2012; 8:4393-4404. [PubMed: 26605601]

22. Lee J, Miller BT, Damjanović A, Brooks BR. J Chem Theory Comput. 2014; 10:2738-2750. [PubMed: 25061443]

23. Lee MS, Salsbury FR Jr, Brooks CL III. Proteins. 2004; 56:738-752. [PubMed: 15281127]

24. Khandogin J, Brooks CL III. Biophys J. 2005; 89:141-157. [PubMed: 15863480]

25. Goh GB, Hulbert BS, Zhou H, Brooks CL III. Proteins. 2014; 82:1319-1331. [PubMed: 24375620]

26. Wallace JA, Shen JK. J Chem Theory Comput. 2011; 7:2617-2629. [PubMed: 26606635]

27. Alexov E, Mehler EL, Baker N, Baptista AM, Huang Y, Milletti F, Nielsen JE, Farrell D, Carstensen T, Olsson MHM, Shen JK, Warwicker J, Williams S, Word JM. Proteins. 2011; 79:3260-3275. [PubMed: 22002859]

28. Shi C, Wallace JA, Shen JK. Biophys J. 2012; 102:1590-1597. [PubMed: 22500759]

29. Brooks BR, et al. J Comput Chem. 2009; 30:1545-1614. [PubMed: 19444816]

30. MacKerell AD Jr, et al. J Phys Chem B. 1998; 102:3586-3616. [PubMed: 24889800]

31. MacKerell AD Jr, Feig M, Brooks CL III. J Am Chem Soc. 2004; 126:698-699. [PubMed: 14733527] 
32. Vanommeslaeghe K, Hatcher E, Acharya C, Kundu S, Zhong S, Shim J, Darian E, Guvench O, Lopes P, Vorobyov I, MacKerell AD Jr. J Comput Chem. 2010; 31:671-690. [PubMed: 19575467]

33. Milletti F, Storchi L, Sforna G, Cruciani G. J Chem Inf Model. 2007; 47:2172-2181. [PubMed: 17910431]

34. Shalaeva M, Kenseth J, Lombardo F, Bastin A. J Pharm Sci. 2008; 97:2581-2606. [PubMed: 18228610] 

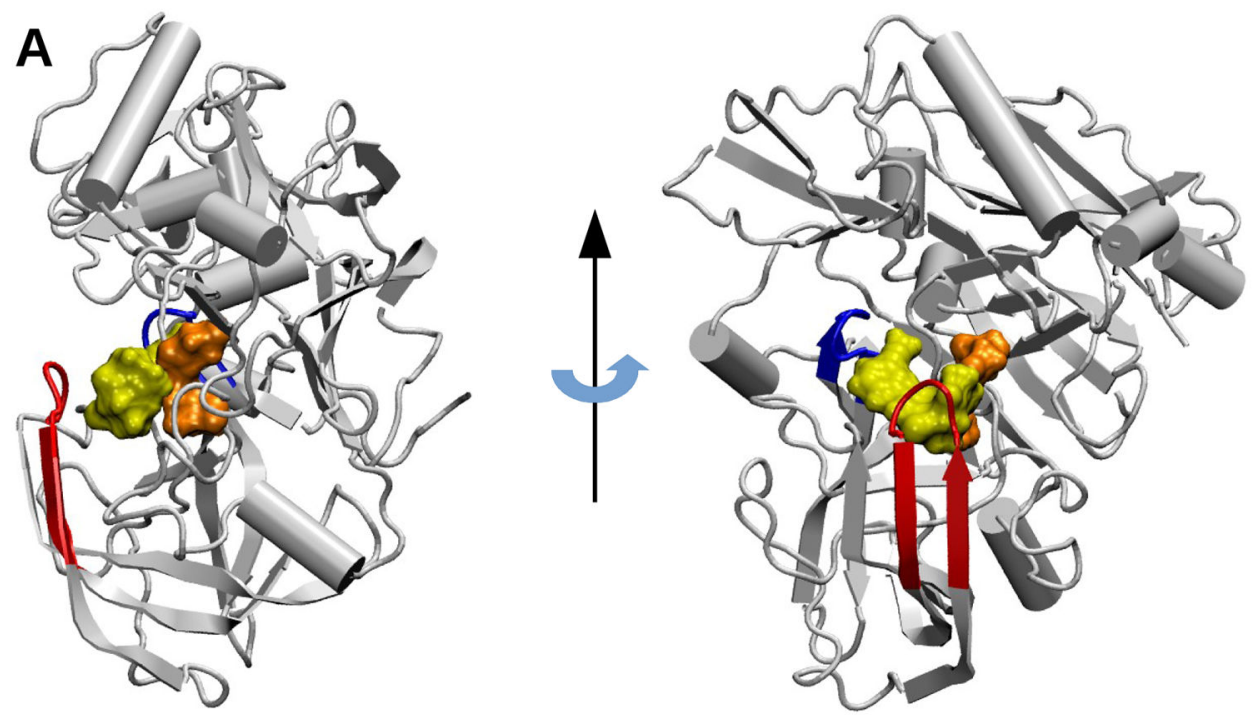

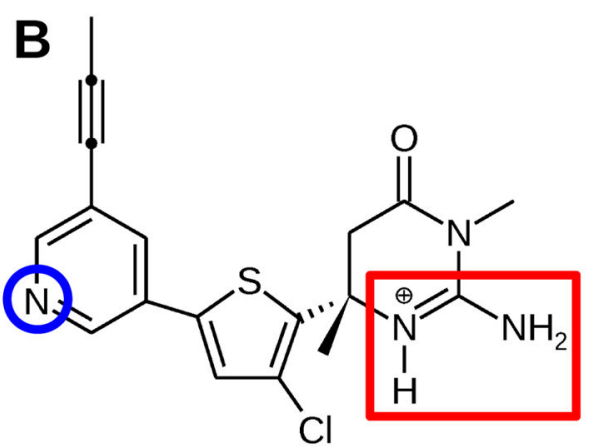

1

$\mathrm{IC}_{50}: 11 \mathrm{nM}$

$\mathrm{pK}_{\mathrm{a}}: 7.5$
C

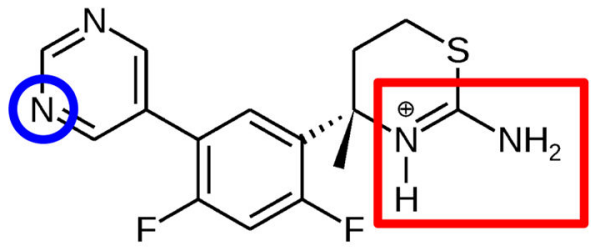

2

$\mathrm{pK}_{\mathrm{a}}: 8.7$

Figure 1.

Structures of BACE1 and the two small-molecule inhibitors. A. BACE1 in complex with inhibitor 1 (PDB ID 4FRS ${ }^{7}$ ). The inhibitor, catalytic dyad, flap, and 10s loop are shown in yellow, orange, red, and blue, respectively. B. Iminopyrimidinone-based inhibitor 1 discovered by Merck (MK8698). ${ }^{7}$ C. Aminothiazine-based inhibitor $\mathbf{2}$ discovered by Eli Lilly (LY2811376). ${ }^{8}$ The two endo- and exocyclic amines on the ABM are highlighted by a red rectangle. The nitrogen on the pyridine/pyrimidine ring (titratable in the simulations) is indicated by a blue circle. Experimental cell $\mathrm{IC}_{50}$ values for $\mathbf{1}$ and $\mathbf{2}$ are taken from ref 7 and ref 8, respectively. Experimental $\mathrm{p} K_{\mathrm{a}}$ 's refer to those of the endocyclic amine group of the ABM: $\mathbf{1}$ is taken from ref ${ }^{7}$, while $\mathbf{2}$ was obtained by capillary electrophoresis experiments (see Methods and Protocols). 

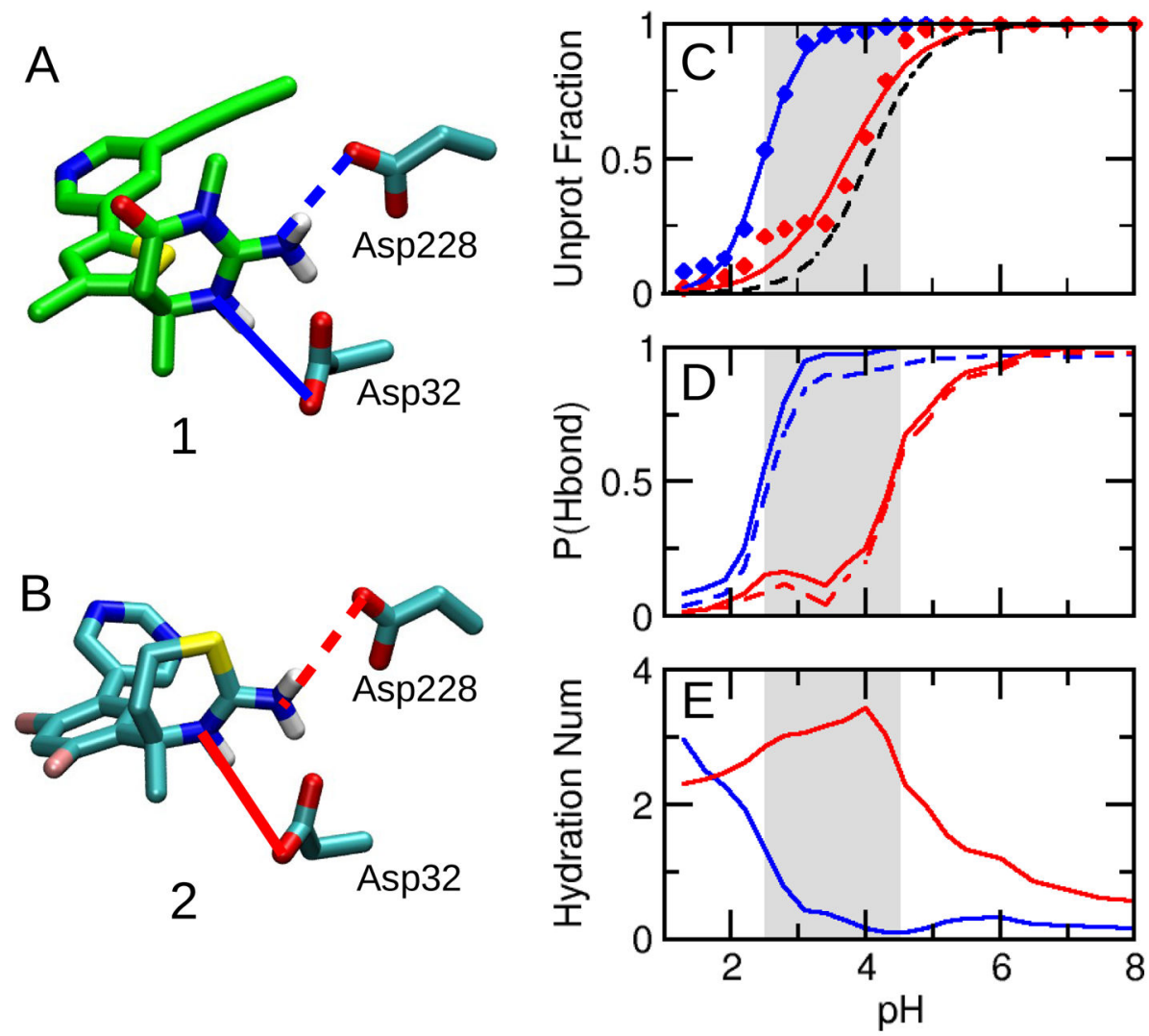

Figure 2.

Titration of the BACE1 catalytic dyad affects the interaction with inhibitor. $\mathbf{A}$ and $\mathbf{B}$. Inhibitors $\mathbf{1}$ and $\mathbf{2}$ interacting with the catalytic dyad. The solid and dashed lines depict hydrogen bonds with Asp32 and Asp228, respectively. C. Unprotonated fractions of Asp32 at different $\mathrm{pH}$. Simulations of BACE1 complexed with $\mathbf{1}$ and $\mathbf{2}$ are shown in blue and red, respectively. The curves are the best fits to the Hill equation. The black dashed curve represents the apo BACE1 simulation. The simulated active $\mathrm{pH}$ range of apo BACE1 is indicated by the grey area. ${ }^{5}$ D. pH-dependent occupancy of the hydrogen bonds with Asp32 (solid) and Asp228 (dashed). A hydrogen bond was considered to be present if the heavy atom donor-acceptor distance was below $3.5 \AA$ and the acceptor-donor-H angle was less than $30^{\circ}$. E. pH-dependent hydration number of Asp32. Hydration number refers to the number of water molecules within the first solvation shell, defined as any water oxygen within $3.5 \AA$ of the carboxylate oxygens of Asp. 

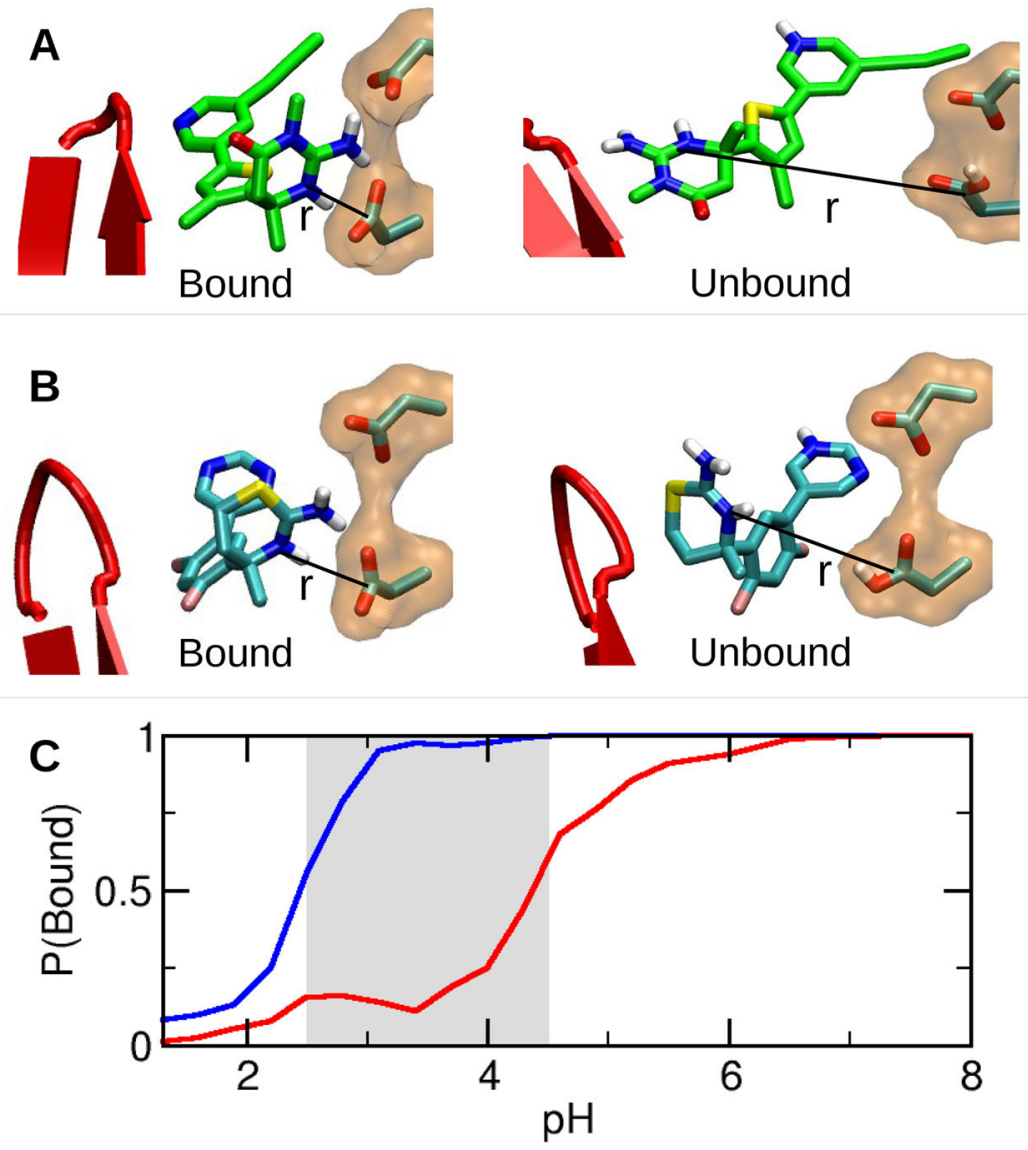

Figure 3.

$\mathrm{pH}$-dependent binding of each inhibitor to BACE1. A. A bound configuration of 2 with BACE1. B. An unbound configuration of 2 with BACE1. C. Population of the bound configurations as a function of $\mathrm{pH}$. The simulations in the presence of $\mathbf{1}$ and $\mathbf{2}$ are shown in blue and red, respectively. 

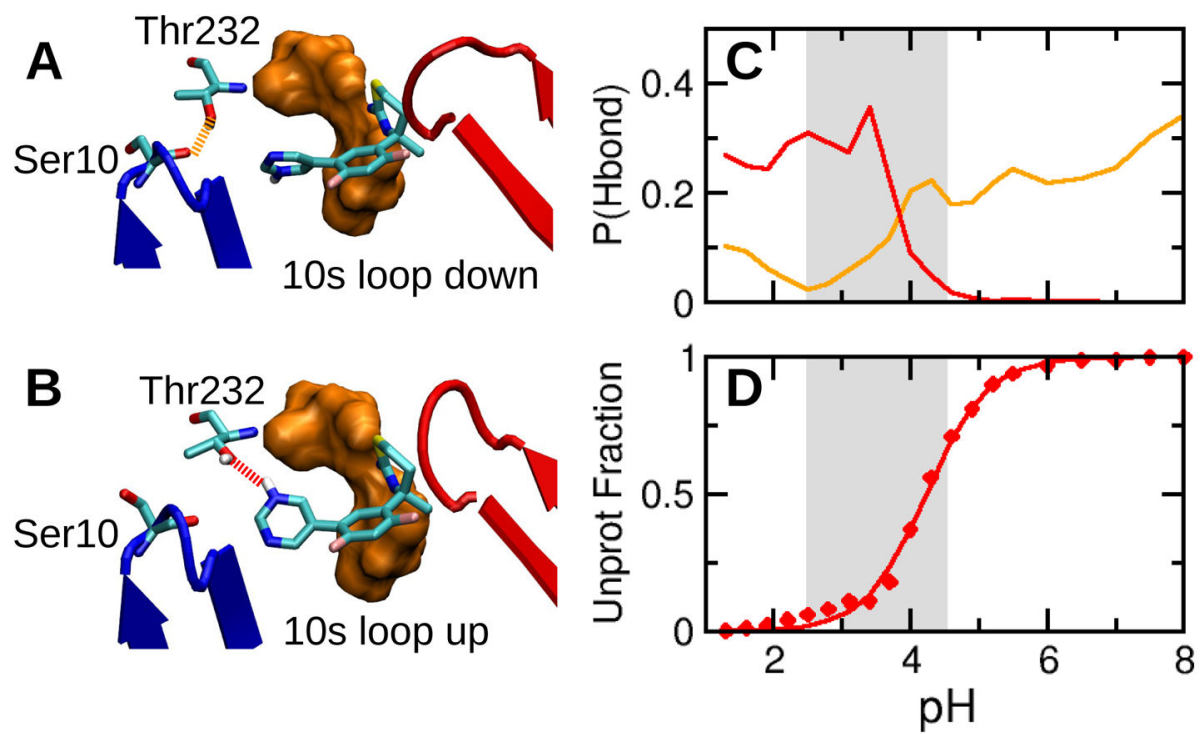

Figure 4.

Inhibitor 2 competes with the 10s loop for the interaction with Thr232. A. Binding mode of 2 at high $\mathrm{pH}$. The 10s loop and flap are shown in blue and red, respectively. Ser10 forms a hydrogen bond with Thr232, and the 10s loop is down. B. Binding mode of 2 at low pH. A hydrogen bond is formed between Thr232 and the pyrimidine nitrogen of $\mathbf{2}$, and the 10s loop is up. C. pH-dependent hydrogen bond occupancy. The hydrogen bond between Thr232 (hydroxyl) and Ser10 (backbone carbonyl) is shown in orange, while that between Thr232 (hydroxyl) and the pyrimidine nitrogen of $\mathbf{2}$ is shown in red. D. Unprotonated fraction of the pyrimidine nitrogen of $\mathbf{2}$ at different $\mathrm{pH}$. The solid curve is the best fit to the Hill equation. 